Techniques \& Culture

Revue semestrielle d'anthropologie des techniques

$57 \mid 2011$

Geste et Matière

\title{
Notes sur le calendrier japonais
}

André Leroi-Gourhan

\section{(2) OpenEdition}

Journals

Édition électronique

URL : https://journals.openedition.org/tc/5785

DOI : $10.4000 /$ tc. 5785

ISBN : 1952-420X

ISSN : 1952-420X

Éditeur

Éditions de l'EHESS

Édition imprimée

Date de publication : 15 décembre 2011

Pagination : 46-53

ISBN : 978-2-7351-1437-5

ISSN : 0248-6016

Référence électronique

André Leroi-Gourhan, « Notes sur le calendrier japonais », Techniques \& Culture [En ligne], 57 | 2011, mis en ligne le 30 juin 2012, consulté le 29 septembre 2022. URL : http://journals.openedition.org/tc/5785 ; DOI : https://doi.org/10.4000/tc.5785 


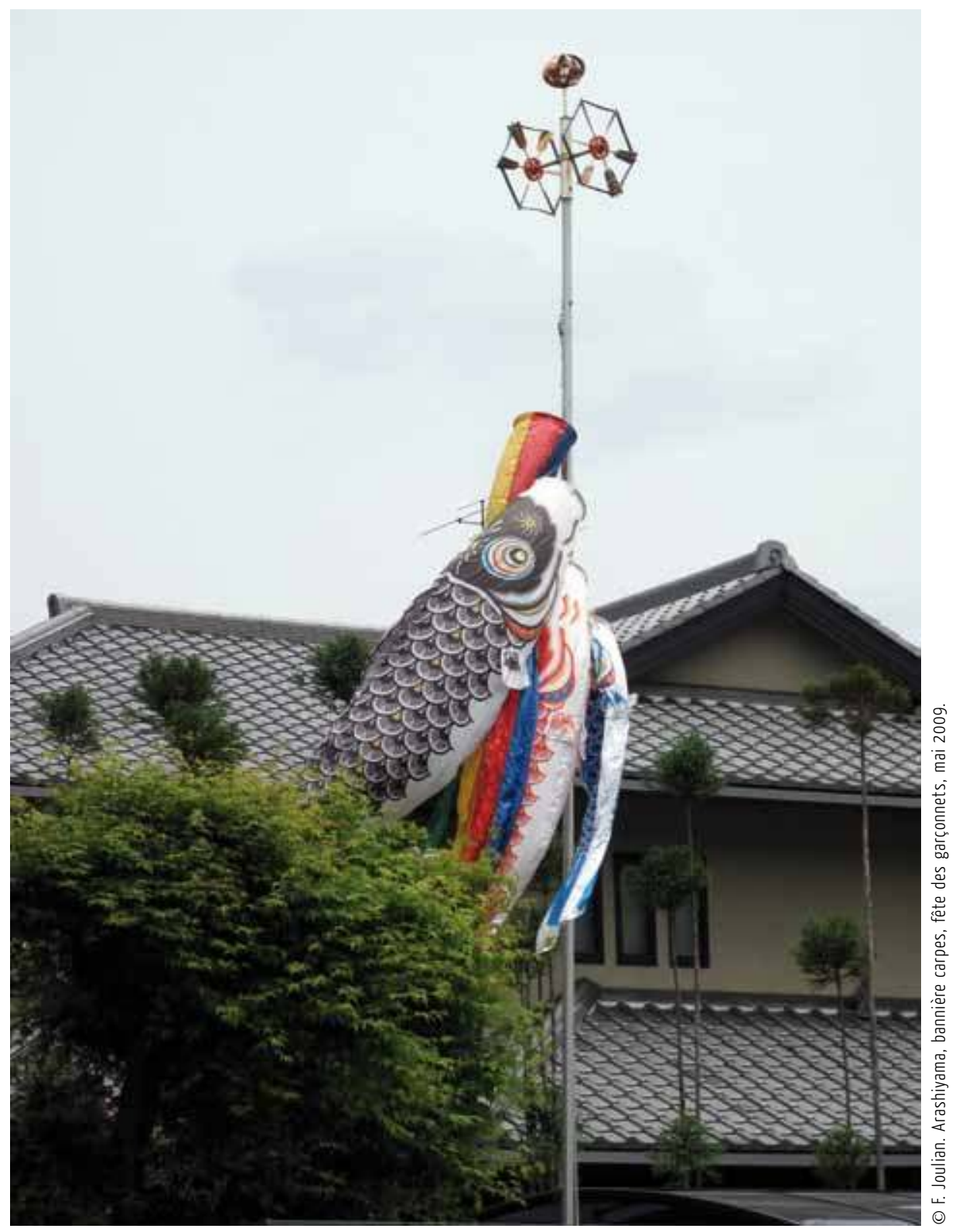




\section{NOTES SUR LE CALENDRIER JAPONAIS}

Cet article, simple mise en ordre de notes prises sur le terrain, ne prétend pas donner un tableau complet du calendrier japonais mais en tracer quelques unes des grandes lignes.

Une première réflexion s'impose devant les faits : qu'il s'agisse du calendrier des fêtes bouddhistes ou shintoïstes, tout se rassemble autour des quatre points culminants de l'année astronomique : équinoxe de printemps, solstice d'été, équinoxe d'automne, solstice d'hiver. Les fêtes des solstices sont, de la manière la plus nette, des fêtes où le feu joue un rôle important.

Cette esquisse ouvre de si larges horizons à la comparaison qu'on peut se demander si elle présente un réel intérêt. En effet il n'est guère de calendrier des rites, y compris le nôtre, qui ne soit rythmé à quatre temps par le mouvement de l'année. Des feux de la $\mathrm{S}^{\mathrm{t}} \mathrm{Jean}$ à la Bûche de Noël, presque tous les peuples ont leurs feux d'été et d'hiver. Nous considérons donc comme une banalité indispensable de dire que le Japon ne fait pas exception à la règle, il suit le rythme des saisons, lie la fécondité du sol à celle de la famille et le retour des âmes ancestrales aux rites des accordailles; ses pratiques sont chargées de symboles naifs et lorsque l'année se termine il liquide, avec les balayures et les vieilles amulettes, ses maux abandonnés au feu ou au courant de la rivière.

Mais quand on entre dans la réalité des faits, deux impressions presque contradictoires se dégagent : la première est qu'il existe plusieurs calendriers, plusieurs traditions qui se superposent avec de nombreux porte-à-faux; la seconde est que la masse populaire s'accommode de ces systèmes variés et en dégage un cycle d'apparence homogène.

Nous évoquerons ici, tout d'abord, le calendrier dit « impérial », pratiqué au Palais et dans différents grands temples. Imposé progressivement à la masse depuis trois quarts de siècle il apparaît, malgré tout, comme un ajustage assez factice : 
2 janvier : fête du sacrifice aux origines de l'empire,

11 février : fête de la fondation,

17 février : fête des moissons à venir,

Équinoxe de printemps: sacrifice aux ancêtres impériaux,

Solstice d'été : fêtes pour les ancêtres impériaux récents,

Équinoxe d'automne : sacrifice aux ancêtres impériaux,

ler octobre : fête des prémices,

23 novembre : fête du premier riz,

Solstice d'hiver : danses sacrées du sanctuaire impérial.

Cet extrait permet de dégager l'hétérogénéité du calendrier impérial. La fête du 2 janvier, correspondant au Nouvel-An chrétien, est un ajustage récent, imposé par le changement de calendrier. La fête du 11 février correspond à l'ancien nouvel an et coïncide avec les rites populaires d'expulsion de l'année écoulée ; un ajustage ancien en fait l'anniversaire de la fondation de chaque règne.

La suite recouvre deux cycles distincts : tout d'abord, les fêtes agricoles du 17 février, $1^{\text {er }}$ octobre et 23 novembre où l'Empereur délie, suivant les traditions chinoises, les opérations de la Terre : ouverture de l'année agricole, prémices; d'autre part le cycle à quatre temps du culte aux ancêtres impériaux qui est simplement lié à la famille impériale et correspond aux fêtes que chaque famille consacre à ses propres ancêtres. Il se termine au solstice d'hiver par les danses de fin d'année et les feux qu'on retrouve dans tout l'Extrême-Orient.

Ce premier calendrier, par l'effet de la restauration du pouvoir impérial en 1868, s'est trouvé progressivement imposé à la pratique populaire. Celle-ci, plutôt shintoïste ou plutôt bouddhiste, n'est jamais exclusive et montre un réel éclectisme. Les cloisons confessionnelles sont très lâches et l'on peut parler, pour la majorité des sujets pratiquants, d'un syncrétisme véritable. Le mélange a été rendu inévitable par la pratique obligatoire du shintoïsme impérial, fortement contaminé au viie-viiie siècle par le bouddhisme continental et par l'existence d'une foule de pratiques esthétiques, d'inspiration plutôt shintoïste, mais suivies depuis longtemps par tout le monde. Une certaine concurrence a joué aussi et shintoïsme comme bouddhisme ont fini par acquérir des fêtes-répliques, à des dates communes ou très voisines.

Dans l'ensemble l'année religieuse tient encore au vieux calendrier et débute en Février.

FÉVRIER : Setsu-bun saï : Fête d'expulsion des vertus périmées.

A) Shintô (observée au temple de Yoshida, à Kyôto, 2 février). Deux démons, rouge et vert, tournent trois fois autour du pavillon de danse, poursuivis à grands cris par l'exorciste, le visage couvert d'un masque rouge à quatre yeux et armé d'un trident. Après le départ des démons les archers tirent des flèches de roseau avec des arcs de pêcher. La foule se dispute les flèches.

On dépose les enveloppes-amulettes de l'année écoulée dans un cercle de pierres où elles sont incinérées et on achète de nouvelles enveloppes et des sachets de haricots.

B) Bouddhisme (observée au Rozan-ji, à Kyôto, 3 février).

Trois démons, rouge, vert et noir, tournent trois fois dans le sens solaire autour d'une montagne de branches vertes. Ils pénètrent dans le temple à grands cris. Deux bonzes armés d'arcs et deux autres portant chacun un plat de haricots torréfiés se postent sur la galerie. Les démons sortent en hurlant sous les flèches et les jets de haricots que le public se partage avec passion. Les bonzes laissent choir les plats vides dont les enfants se partagent les miettes.

De fin Février en Avril, suivant les lieux : fêtes pour délier la terre (Dogesai) et reprise des travaux agricoles. 
MARS : Ohina matsuri : Fête des poupées (fête des fillettes).

Suivie sans distinction de confession, d'inspiration plutôt shintoïste. Des poupées, figurant un empereur et une impératrice, entourées de dames de la cour et de musiciens sont groupées sur les gradins d'une petite estrade. Divers objets comprenant des poupées de caractère franchement religieux et les poupées-jouets sont disposés sur les bas gradins. Les fillettes de la maison reçoivent leurs amies devant l'estrade disposée dans la pièce de réception.

\section{MAI : Shobu-saï : Fête des iris (fête des garçonnets).}

Dans la maison une armure (ou un modèle réduit d'armure) est placée dans l'alcôve d'honneur. Bouquets d'iris dont les feuilles symbolisent la lame du sabre et la droiture du guerrier, carpe peinte symbolisant l'énergie et la ténacité. Les garçonnets prennent un bain de feuilles d'iris.

Dans quelques grands temples, défilés d'enfants armés et courses de chevaux. Le cheval gagnant communique sa force aux ex-voto peints sur des planchettes et vendus aux fidèles.

Ces deux fêtes de printemps assurent le développement des qualités morales des enfants.

À la même saison, dans les temples shintoïstes et bouddhistes, pantomimes et représentations théâtrales. Les pièces sont jouées par des fidèles; chez les shintoïstes elles figurent fréquemment la légende du dieu local ; chez les bouddhistes des épisodes à tendance moralisatrice empruntés au même fonds que le théâtre de Nô.

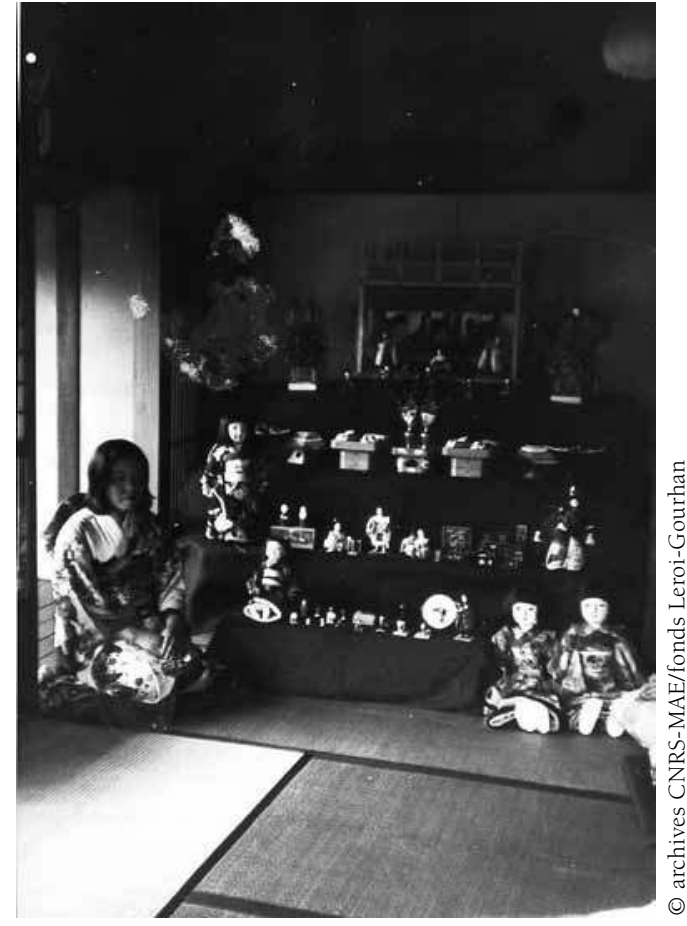

Fête des poupées Autel.

Fête des poupées Fillettes devant l'autel des poupées. danses et des chants alternés des garçons et des filles qui procèdent en musique au repiquage de la rizière du temple. Cette fête est dédiée à Inari, divinité du riz, et à ses renards blancs.

Agata matsuri: Fête d'Agata.

Proscrite, mais encore pratiquée dans quelques temples. Procession dans l'obscurité au cours de laquelle la licence totale est permise aux participants et aux spectateurs. Liée à la fertilité et à la moisson à venir.

Take kiri : Fête des bambous (observée au temple bouddhiste de Kurama, au nord de Kyôto).

Les paysans de Tango et Tanba se livrent à une joute en tronçonnant deux gros bambous à coups de sabre. Les bambous figurent deux dragons. Le parti vainqueur est assuré de la meilleure récolte.

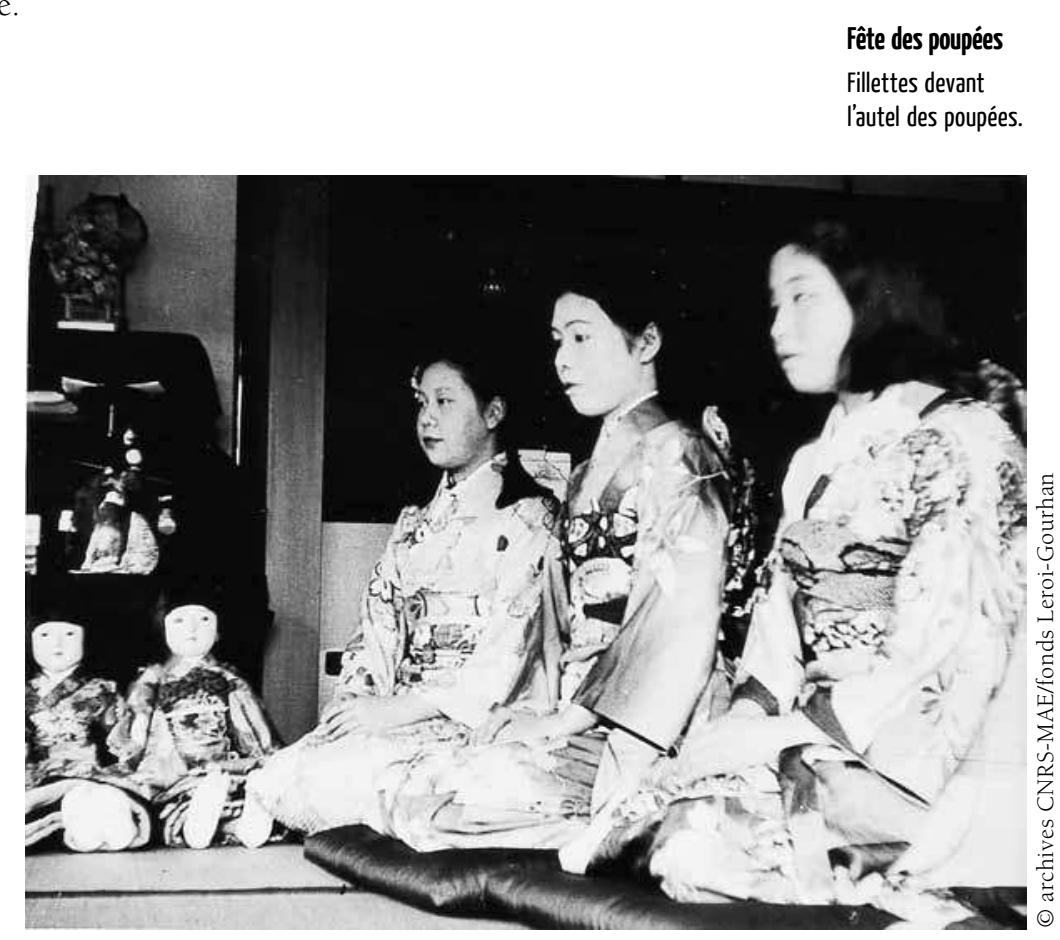




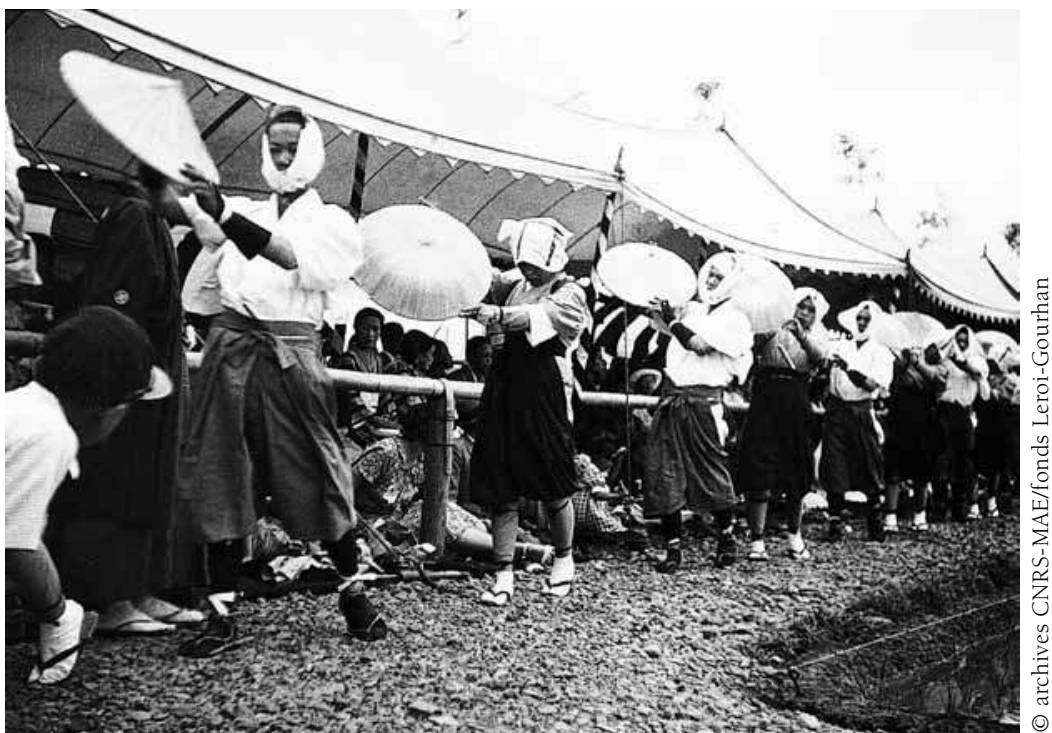

Mukômachi. Fête du repiquage du riz, le 19 juin 1938

Danseurs et danseuses.
JUILLET : Mitarashi : Fête de purification.

Pratiquée par les shintoïstes et les bouddhistes. À Kyôto, au temple de Gion, purification des épidémies avec défilé dans la ville de chars géants, d'inspiration indienne, traînés par des centaines de fidèles. Comme aux Indes, acrobaties au sommet des mâts de chars avec des accidents fréquents.

AOÛT : Bon-odori : Danses du bon.

Ensemble complexe de cérémonies liées à l'arrivée, au séjour et au départ des âmes des ancêtres de la famille. Shintoïste et bouddhiste. L'épisode central consiste en danses et chants alternés des garçons et des filles fréquemment suivis de licence. Dans les districts du sud, parallélisme frappant avec les fêtes d'été de la Chine ancienne et des Barbares actuels de l'Indochine du Nord.

L'épisode du départ des âmes est marqué par l'abandon de lanternes flottantes, de bateaux miniatures et de poupées au courant des rivières (fête des lanternes).

SEPTEMBRE : Shishi no maï : danse des lions.

Dans les rues et les temples; à dates variables, ont lieu des danses masquées : tantôt celle de deux lions qui jouent, tantôt celle du lion ou du dragon combattu par un guerrier. Elles semblent correspondre à des pratiques chinoises aux équinoxes.

OCTOBRE-NOVEMBRE : Suivant les lieux,

Shuki daï-saï : grande fête de l'automne, liée à la moisson du riz et aux prémices de la récolte.

DÉCEMBRE : Une suite importante de fêtes d'hiver occupe la fin de décembre et le début de janvier. Le feu y intervient, avec l'eau, de façon constante.

O Kagura: danses sacrées.

Exécutées dans les temples shintoïstes, nous en citons deux exemples caractéristiques

On matsuri : La grande fête (au temple de Kasuga, à Nara).

16 et 17 décembre : dans la nuit, à la lueur de brasiers. Sur le tertre devant le reposoir du dieu du temple, danses masquées représentant les vestiges de la danse chinoise antique des douze animaux de l'année. Danse du dragon (Ran-ryû ô), du cheval (batô), du phénix (shin tori-no), du porc (Nasori).

18 - 19 décembre : représentations de théâtre classique (Nô), illustrant des légendes relatives aux divinités; courses de chevaux et luttes.

Hana-matsuri : fête des fleurs (dans la préfecture d'Aichi).

Les dieux sont invités à prendre place sur une montagne (cadre suspendu au plafond) et sur quatre arbustes plantés aux points cardinaux. Un chaudron d'eau bouillante est placé au centre. 
Deux démons agitant des torches sont expulsés après une danse très brève accompagnée de luttes et d'aspersion des spectateurs à l'aide de balais trempés dans l'eau chaude.

O mizu tori : prendre l'eau sacrée. Observée à Nara, au [Niga]tsu dô (bouddhiste) [du $1^{\text {er }}$ au 14 mars].

Les bonzes tournent pendant des heures autour du sanctuaire. Ils jonglent avec une torche géante puis vont chercher l'eau nouvelle, à l'aube, dans le puits du sanctuaire.

Kemari : football rituel.

Joué au palais impérial par des nobles de la cour. Sur un terrain carré aux angles duquel sont plantés le cerisier du printemps (Est), le saule de l'été (Sud), l'érable de l'automne (Ouest) et le pin de l'hiver (Nord). Un ballon de cuir de cheval figurant le soleil est lancé au pied par les joueurs et décrit des cercles dans le sens des aiguilles de la montre. Survivance d'un rituel chinois attesté sous les Han (II siècle avant notre ère).

Ces quelques indications calendériques prêteraient à de longs développements sur l'origine des rituels japonais et sur le symbolisme des fêtes. Nous avons, au début de cet article, posé que le Japon s'intègre dans l'ensemble, pratiquement mondial, des fêtes saisonnières. Si les rapprochements lointains, nous semblent assez précaires, il est par contre assez facile de démêler les principales sources de ces fêtes. Comme dans tous les domaines, le Japon apparaît extraordinairement conservateur, on pourrait presque dire « collectionneur ». Il superpose, sans élaboration profonde, toutes les pratiques que deux mille ans de vie religieuse lui ont apportées de l'extérieur.

La vague la plus ancienne paraît être chinoise et le Shintô antique a emprunté, probablement par la Corée, les premiers cadres de son culte officiel.

La seconde vague apporte à la fois au shintoïsme les cérémonies ou les costumes de la cour chinoise des T'ang, au VII ${ }^{e}-\mathrm{VIII}^{\mathrm{e}}$ siècle, et les pratiques du bouddhisme indien, tamisé par la Chine. Il en est resté, du côté shintoïste, tout le cadre des rituels de cour, sacrifices aux ancêtres, jeux de ballon, danses d'hiver. Du côté bouddhiste il est resté les cérémonies liturgiques, peu contaminées et en quelque sorte en marge de la pratique populaire. Du mélange Chine-Inde, Shintô-Bouddhisme, sont nées des cérémonies complexes : le très vieux fonds des danses alternées de paysans et paysannes s'accommode des cérémonies au temple bouddhiste du lieu, les bonzes chassent les démons avec l'arc de bois de pêcher de la Chine, les chars de l'Hindouisme s'associent au dieu cheval du Shintô et les danses de lions sont exécutées dans tous les temples.

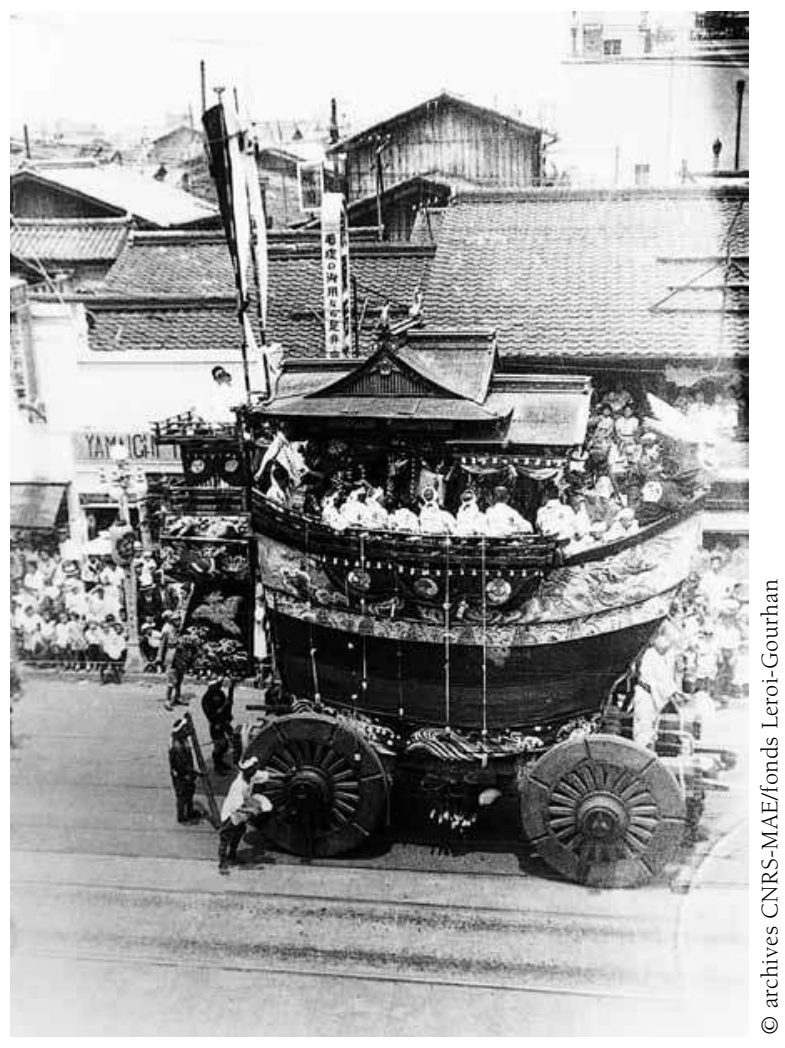

Gion Matsuri : fête de Gion Char.

Sous toutes ces pratiques existent certainement des éléments propres au Japon ancien, mais celui-ci nous est pratiquement inconnu. Les textes du vil siècle sont muets sur le calendrier rituel et nous laissent seulement des noms de dieux qui sont morts ou qu'on a ressuscités de date encore fraîche. Au moment où il assimile l'écriture il reçoit du même coup le calendrier chinois et les textes sanscrits. Tout cela, par la suite, est devenu millénaire et japonais, mais les seuls témoins très anciens auxquels on puisse se référer sont tous des témoins continentaux. 


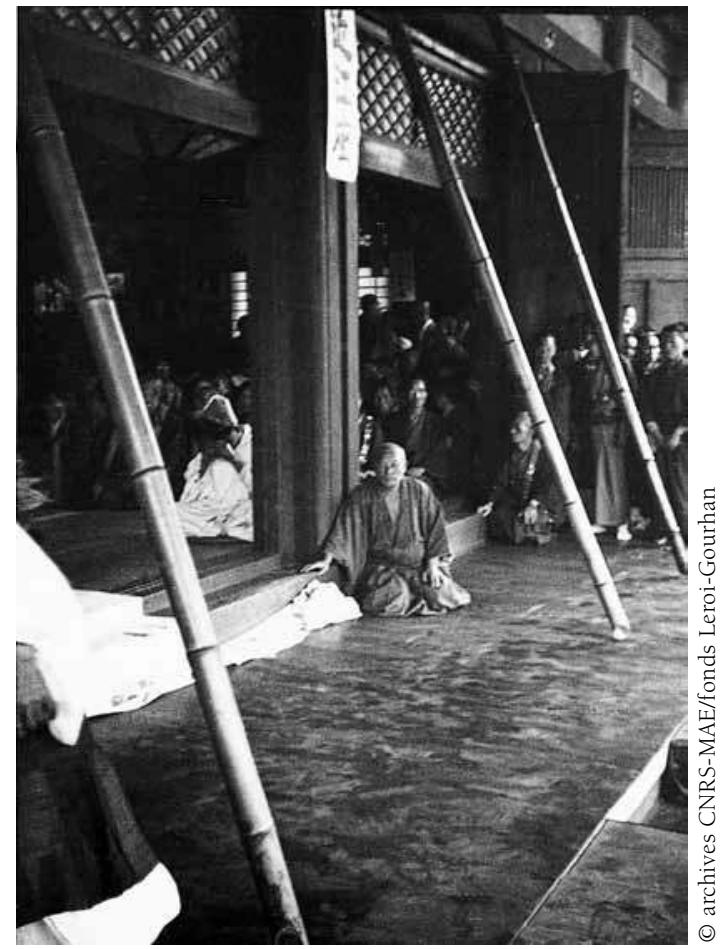

Bambous en position avanrt le concours.

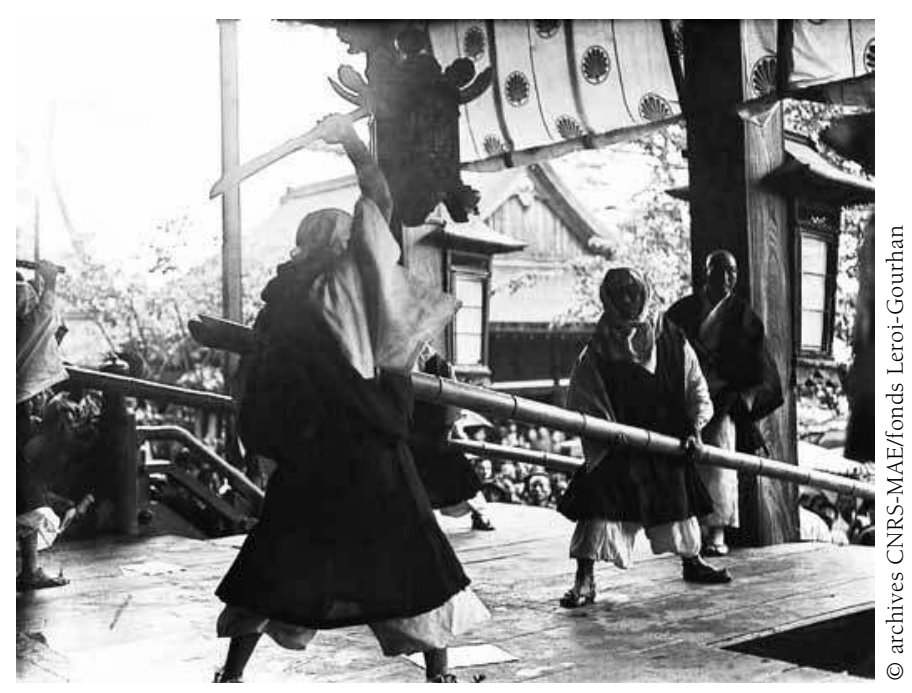

La coupe.

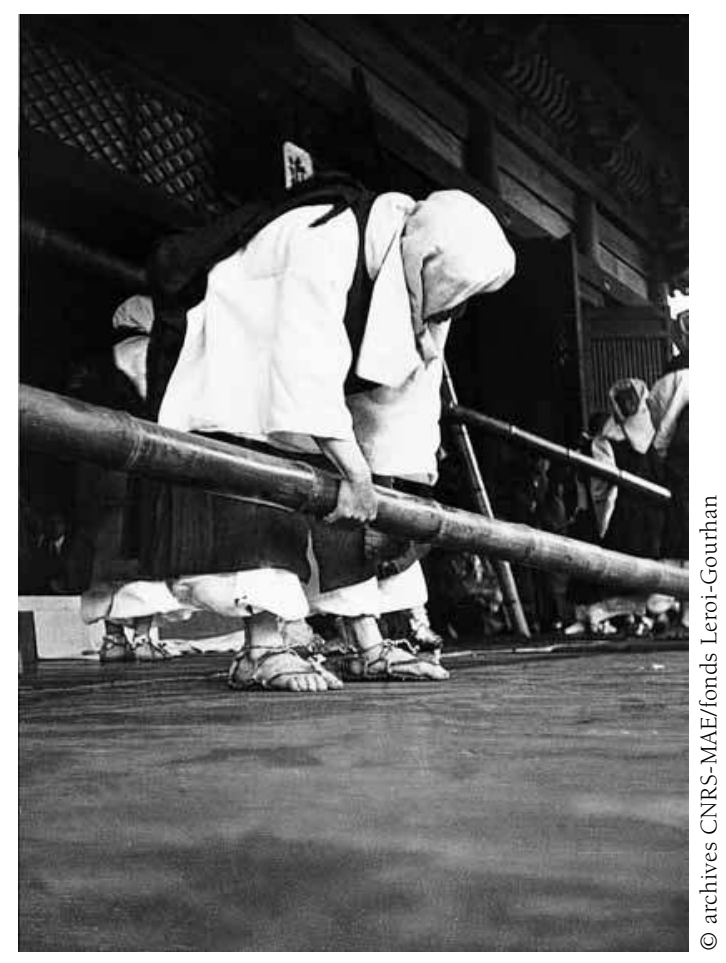

Mise en place d'un bambou.

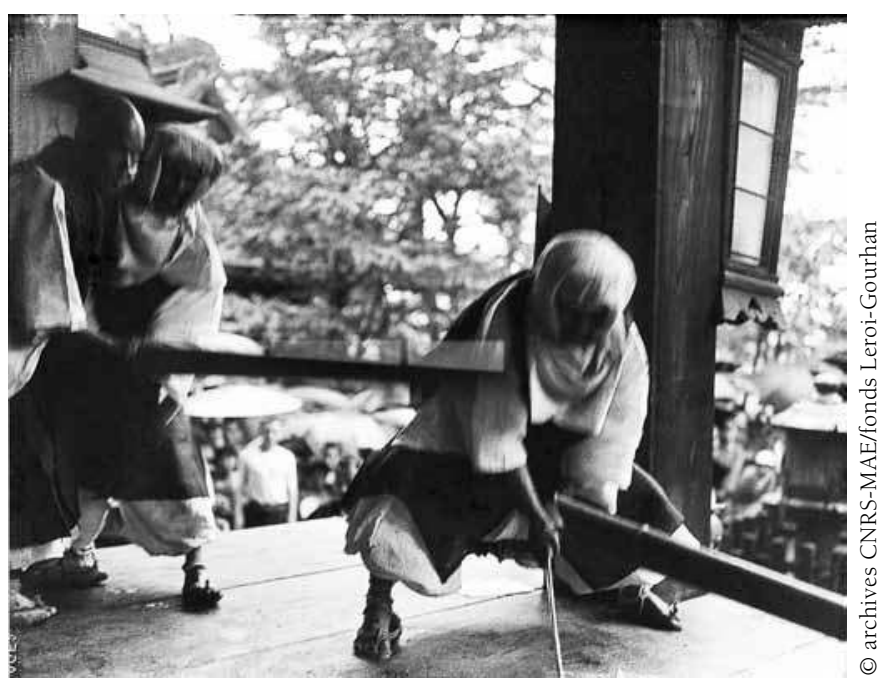

La coupe. 
Arashiyama, football traditionnel

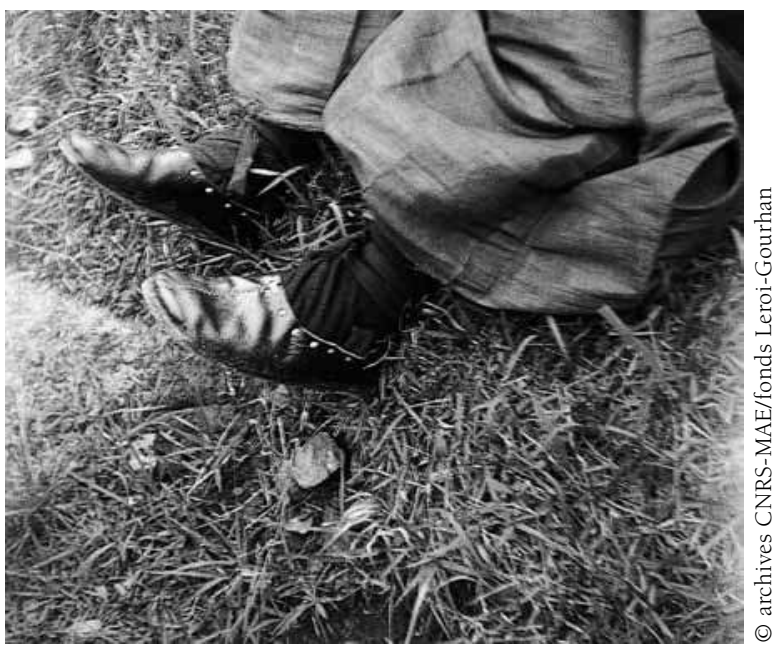

Mocassins des joueurs.

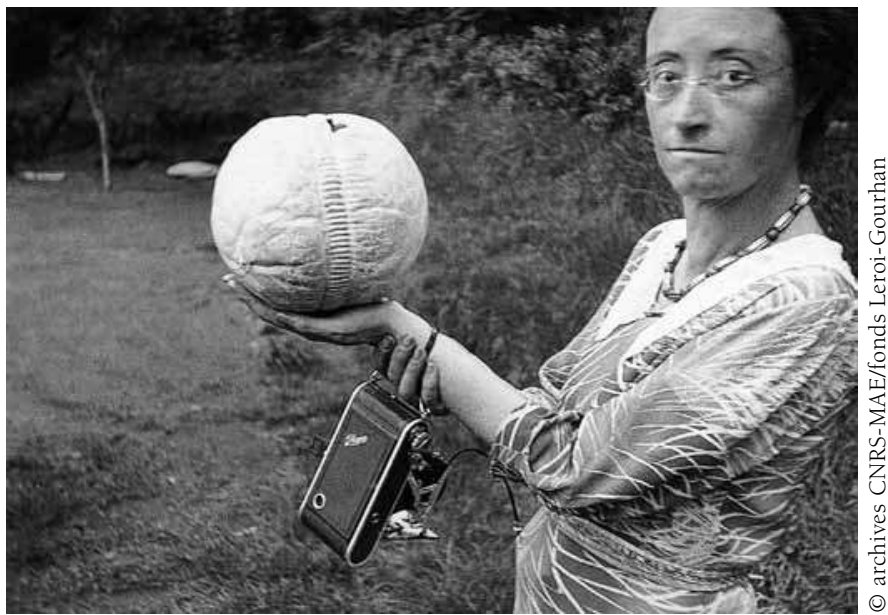

Ballon, et Rose Houyoux.

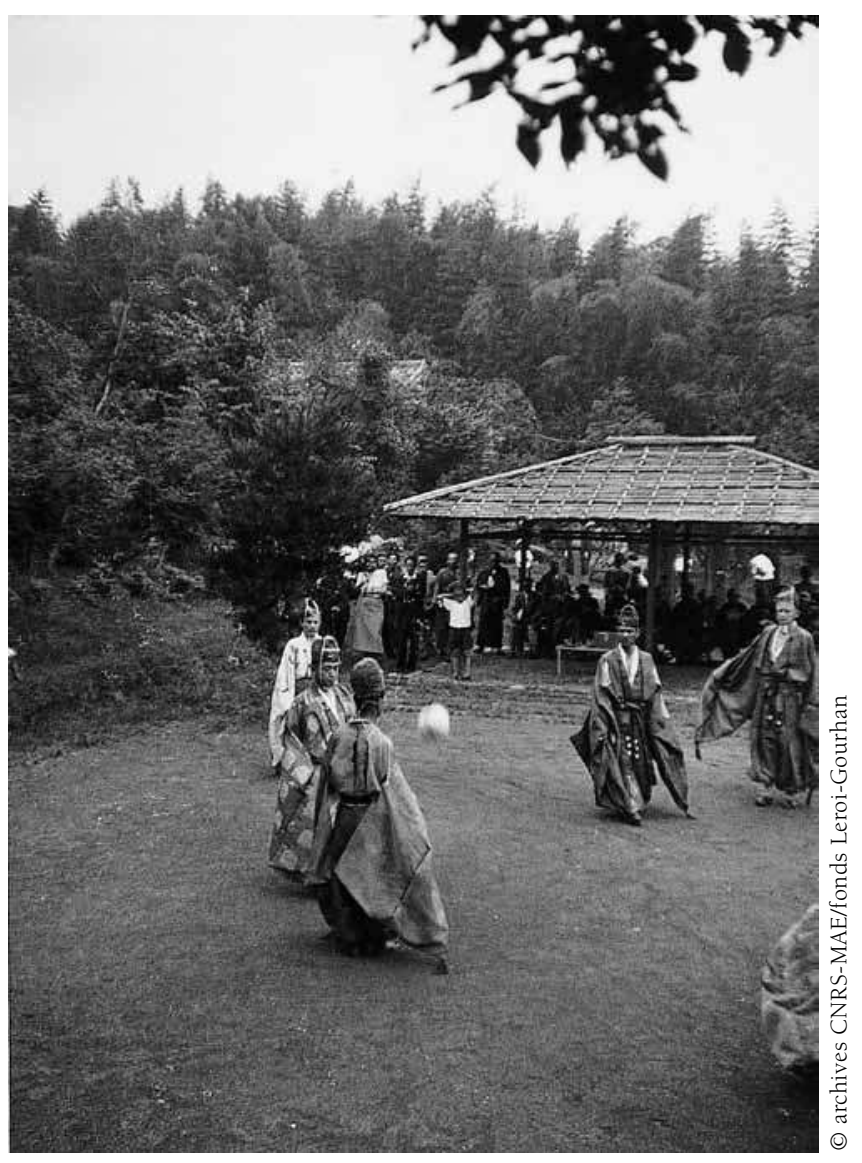

Partie, vue du sud.

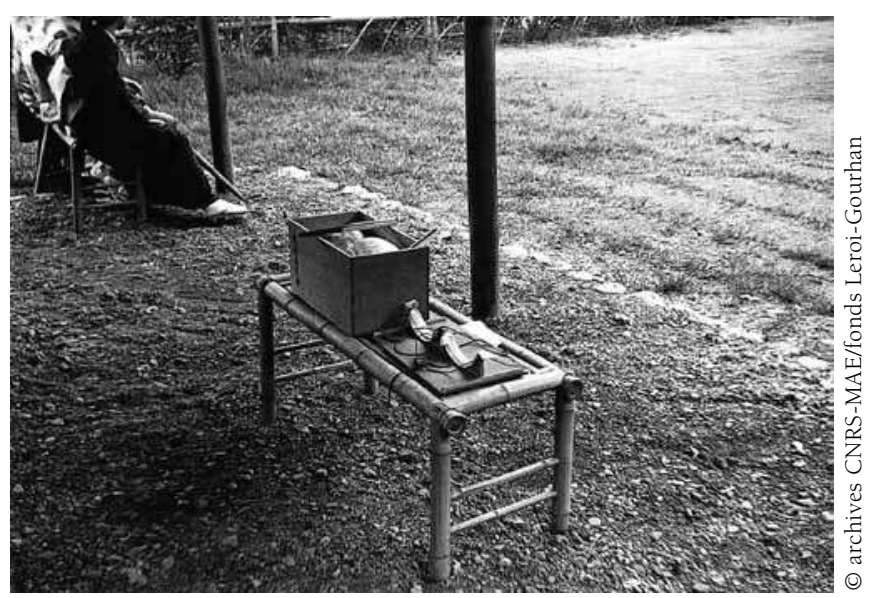

Sous le kiosque, ballon dans sa boîte, support des ballons de jeu. 